\title{
Seven Cool Number Patterns
}

\author{
Mohammed R. Karim \\ Alabama A\&M University, Normal, Alabama, USA \\ mohammed.karim@aamu.edu
}

\begin{abstract} particular number. An interpretation of the inspiring number pattern is also provided.

Keywords: Number Patterns, Transpose, Packing.

\section{Introduction}

$$
\begin{aligned}
1 \times 8+1 & =9 \\
12 \times 8+2 & =98 \\
123 \times 8+3 & =987 \\
1234 \times 8+4 & =9876 \\
12345 \times 8+5 & =98765 \\
123456 \times 8+6 & =987654 \\
1234567 \times 8+7 & =9876543 \\
12345678 \times 8+8 & =98765432 \\
123456789 \times 8+9 & =987654321
\end{aligned}
$$
\end{abstract}

Inspired by a number pattern found while reading an online newspaper seven number patterns are created. Behind each pattern there is a distinct algorithm. Some of these patterns are a sequence of a single or double digit, and the rest are packing ups of one or two digits either one or both sides of a

While reading an online news paper from abroad, I found the following number pattern.

It captured my attention. I was fascinated by this amazing number pattern and trying to interpret it in the following way:

It, to me, defines a function $f$ with the domain $\{1,2,3,4,5,6,7,8,9\}$ and as if, each of these digits occupy a box arranged in a row (Figure 1 ) and correspond another digit in the row by the following rules: 


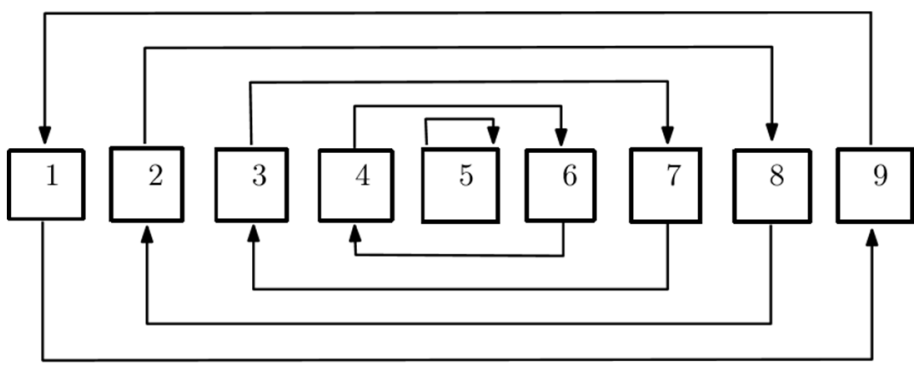

Figure 1

$$
\begin{gathered}
f(1)=[1]^{T}=9 \text { and } f(9)=[9]^{T}=1 \\
f(2)=[2]^{T}=8 \text { and } f(8)=[8]^{T}=2 \\
f(3)=[3]^{T}=7 \text { and } f(7)=[7]^{T}=3 \\
f(4)=[4]^{T}=6 \text { and } f(6)=[6]^{T}=4 \\
f(5)=[5]^{T}=5 \text {, i.e., } 5 \text { corresponds to itself (symmetric). }
\end{gathered}
$$

This means, under this function:

The first box on the left marked by 1 corresponds to the last box on the right marked by 9 .

The second box on the left marked by 2 corresponds the second box on the right marked by 8 .

The third box on the left marked by 3 corresponds the third box on the right marked by 7 .

The fourth box on the left marked by 4 corresponds the fourth box from the right marked by 6 .

The box marked by 5 is in the middle and it corresponds to itself.

Moreover, the last line of the above number pattern shows that

$$
f(123456789)=(123456789)^{T}=987654321,
$$

where $T$ represents the 'Transpose of Digits' similar to the 'Transpose of Matrices' used in Linear Algebra [1]. For example:

$$
(A B)^{T}=B^{T} A^{T},(A B C)^{T}=C^{T} B^{T} A^{T} \text { etc. }
$$

where $A, B, C$ are matrices of appropriate sizes (dimensions) so that the matrix multiplications $A B, A B C$ are defined. Hence one can think of the function $f$ as a function that follows the 'Transposition Rule' as used in Linear Algebra.

Then I was thinking 'Can I create any such number patterns?' The following seven number patterns are the results of the thinking process. 


\section{Number Patterns}

Pattern 1: Having 0, 1, 2, 3, 4, 5, 6 and 7 on the right of 7.

$$
\begin{gathered}
8 \times 8+6=70 \\
87 \times 8+5=701 \\
876 \times 8+4=7012 \\
8765 \times 8+3=70123 \\
87654 \times 8+2=701234 \\
876543 \times 8+1=7012345 \\
8765432 \times 8+0=70123456 \\
87654321 \times 8-1=701234567
\end{gathered}
$$

Pattern 2: Creating Odd Digits $(1,3,5,7,9)$ on the right of 70

$$
\begin{gathered}
9 \times 7+6+10^{0}=70 \\
98 \times 7+5+10^{1}=701 \\
987 \times 7+4+10^{2}=7013 \\
9876 \times 7+3+10^{3}=70135 \\
98765 \times 7+2+10^{4}=701357 \\
987654 \times 7+1+10^{5}=7013579
\end{gathered}
$$

Pattern 3: Creating a Sequence of 9's

$$
\begin{gathered}
(9 \times 9+8)+10^{1}=99 \\
(99 \times 9+8)+10^{2}=999 \\
(999 \times 9+8)+10^{3}=9999 \\
(9999 \times 9+8)+10^{4}=99999 \\
(99999 \times 9+8)+10^{5}=999999 \\
(999999 \times 9+8)+10^{6}=9999999 \\
(9999999 \times 9+8)+10^{7}=99999999 \\
(99999999 \times 9+8)+10^{8}=999999999
\end{gathered}
$$


Pattern 4: Packing up 1's on the Left of 0

$$
\begin{aligned}
1 \times 9+1 & =10 \\
12 \times 9+2 & =110 \\
123 \times 9+3 & =1110 \\
1234 \times 9+4 & =11110 \\
12345 \times 9+5 & =111110 \\
123456 \times 9+6 & =1111110 \\
1234567 \times 9+7 & =11111110 \\
12345678 \times 9+8 & =111111110 \\
123456789 \times 9+9 & =1111111110
\end{aligned}
$$

Pattern 5: Packing up 1's on the Left and 8's on the Right of 0

$$
\begin{gathered}
3 \times 3-1=8 \\
33 \times 33-1=1088 \\
333 \times 333-1=110888 \\
3333 \times 3333-1=11108888 \\
33333 \times 33333-1=1111088888 \\
333333 \times 333333-1=111110888888 \\
3333333 \times 3333333-1=11111108888888
\end{gathered}
$$

Pattern 6: Creating a Sequence of 9's and 0's

$$
\begin{gathered}
9 \times 9-1+10^{1}=90 \\
99 \times 99-1+10^{2}=9900 \\
999 \times 999-1+10^{3}=999000 \\
9999 \times 9999-1+10^{4}=99990000 \\
99999 \times 99999-1+10^{5}=9999900000 \\
999999 \times 999999-1+10^{6}=999999000000 \\
9999999 \times 9999999-1+10^{7}=99999990000000 \\
99999999 \times 99999999-1+10^{8}=9999999900000000
\end{gathered}
$$


Mohammed R. Karim; Seven Cool Number Patterns, Transactions on Networks and Communications, Volume 5 No. 3, June (2017); pp: 16-20

Pattern 7: Packing up 4's and 5's respectively on the Left and Right of 3

$$
\begin{aligned}
6 \times 6-1 & =35 \\
66 \times 66-1 & =4355 \\
666 \times 666-1 & =443555 \\
6666 \times 6666-1 & =4435555 \\
66666 \times 66666-1 & =444355555 \\
666666 \times 666666-1 & =44443555555 \\
6666666 \times 6666666-1 & =4444435555555 \\
66666666 \times 66666666-1 & =444444355555555 \\
666666666 \times 666666666-1 & =44444443555555555
\end{aligned}
$$

\section{ACKNOWLEDGEMENT}

I would like to thank my colleague Dr. Israel Ncube for his help in creating the Figure 1.

\section{REFERENCES}

[1] Poole, David, Linear Algebra-A Modern Introduction (4 ${ }^{\text {th }}$ Edition), Cengage Learning, 2015. 